\title{
The nucleolus and the core-center of multi-sided Bohm-Bawerk assignment markets
}

\section{Journal Article}

\section{Author(s):}

Tejada, Oriol; Nunez, Marina

Publication date:

2012-04

Permanent link:

https://doi.org/10.3929/ethz-b-000048609

\section{Rights / license:}

In Copyright - Non-Commercial Use Permitted

\section{Originally published in:}

Mathematical Methods of Operations Research 75(2), https://doi.org/10.1007/s00186-012-0381-x 


\title{
The nucleolus and the core-center of multi-sided Böhm-Bawerk assignment markets
}

\author{
Oriol Tejada · Marina Núñez
}

Received: 17 June 2010 / Accepted: 8 March 2012 / Published online: 24 March 2012

(C) Springer-Verlag 2012

\begin{abstract}
We prove that both the nucleolus and the core-center, i.e., the mass center of the core, of an $m$-sided Böhm-Bawerk assignment market can be respectively computed from the nucleolus and the core-center of a convex game defined on the set of $m$ sectors. What is more, in the calculus of the nucleolus of this latter game only singletons and coalitions containing all agents but one need to be taken into account. All these results simplify the computation of the nucleolus and the corecenter of a multi-sided Böhm-Bawerk assignment market with a large number of agents. As a consequence we can show that, contrary to the bilateral case, for multisided Böhm-Bawerk assignment markets the nucleolus and the core-center do not coincide in general.
\end{abstract}

Keywords Multi-sided assignment games $\cdot$ Core $\cdot$ Nucleolus $\cdot$ Core-center

\section{Introduction}

The bilateral assignment game was introduced by Shapley and Shubik (1972) as a cooperative model for a two-sided market with transferable utility in which each seller supplies one good and each buyer acquires at most one good in exchange for money.

O. Tejada $(\varangle) \cdot$ M. Núñez

Department of Actuarial, Financial and Economic Mathematics, Universitat de Barcelona, Av. Diagonal, 690, 08034 Barcelona, Spain

e-mail: oriol.tejada@ub.edu

M. Núñez

e-mail: mnunez@ub.edu

O. Tejada

CER-ETH, Center of Economic Research, ETH Zurich, Zürichbergstrasse 18, 8092 Zürich, Switzerland e-mail: toriol@ethz.ch 
Goods may be heterogeneous, and thus a buyer may value differently the goods of different sellers. Shapley and Shubik prove that the core of these games is nonempty and is endowed with a lattice structure. Bilateral assignment games have been widely studied ever since: Solymosi and Raghavan (2001) analyze some properties of the core, whereas Solymosi and Raghavan (1994) and Núñez (2004) prove some results on the nucleolus.

In the present paper we consider a market with an arbitrary finite number of sectors. One sector consists of a finite number of buyers, and each one of the remaining sectors consists of a finite number of sellers. Each seller offers one unit of a good and each buyer demands one bundle formed by exactly one good of each sector. This market can be studied within the framework of multi-sided assignment games, which are introduced by Quint (1991) as the generalization of bilateral assignment games. Unlike these latter games, multi-sided assignment games may have an empty core (Kaneko and Wooders 1982). Multi-sided assignment games have also been studied in Stuart (1997), Sherstyuk (1999) and Tejada and Rafels (2010).

In the aforementioned paper, Shapley and Shubik (1972) also analyze the case of a two-sided market with no product differentiation, known as the bilateral Böhm-Bawerk horse market due to Böhm-Bawerk (1923). For these games it is well known that the core reduces to a segment. Single-valued solutions for the bilateral Böhm-Bawerk assignment game are studied in Núñez and Rafels (2005) who conclude that, without additional information about the bargaining capabilities of the agents, the classical cooperative theory seems to recommend the midpoint of the core segment. This assertion is supported by the fact that, among other single-valued solutions, the nucleolus (Schmeidler 1969) coincides with the midpoint of the core segment, that is, with the mass-center of the core. The mass-center of the core was introduced by GonzálezDíaz and Sánchez-Rodríguez (2007), with the name of core-center, as a single-valued solution for arbitrary coalitional games.

In the case of multilateral markets, the particular case where each buyer places the same valuation on all the bundles is introduced in Tejada (2010) with the name of multi-sided Böhm-Bawerk assignment market. There it is shown that the core of these games is nonempty and it is completely determined by the core of a convex game played by some fictitious players — called sectors — instead of the original agents. This convex game is called the associated sectors game.

The aim of the present paper is to analyze the nucleolus and the core-center of multi-sided Böhm-Bawerk assignment markets. We show that both the nucleolus and the core-center of a multi-sided Böhm-Bawerk assignment market can be respectively computed from the nucleolus and the core-center of the associated sectors game, this being a game with many less players. What is more, only singletons and coalitions containing all agents but one need to be taken into account in the calculation of the nucleolus of this latter game. These results simplify the computation of the nucleolus and the core-center of a multi-sided Böhm-Bawerk assignment market with a large number of agents. As a consequence we can show that, unlike the case of two-sided Böhm-Bawerk markets, the nucleolus does not coincide in general with the core-center in the case of multi-sided Böhm-Bawerk assignment markets.

The structure of the paper is as follows. The preliminaries on coalitional games and multi-sided Böhm-Bawerk assignment games are presented in Sect. 2. In Sect. 3 
we determine which coalitions are to be taken into account for the computation of the nucleolus of a multi-sided Böhm-Bawerk assignment game and we also show that its nucleolus can be obtained from the nucleolus of the related sectors game. Section 4 establishes a parallel result for the core-center. An example is used throughout the paper to illustrate both the model and the results.

\section{Preliminaries and notation}

A coalitional game (a game) is a pair $(N, v)$, where $N$ is the nonempty finite set of players and, for all $S \subseteq N, v(S) \in \mathbb{R}$ is the worth that coalition $S$ can obtain without the cooperation of agents in $N \backslash S$, with $v(\varnothing)=0$. Let $|S|$, or alternatively $s$, denote the cardinality of coalition $S \subseteq N$. As usual, we denote by $2^{N}$ the set of all subsets of $N$ and we use the notation $x(S)=\sum_{i \in S} x_{i}$ and $x(\varnothing)=0$. An imputation is a payoff vector $x \in \mathbb{R}^{N}$, where $x_{i}$ stands for the payoff to player $i \in N$, that is efficient, i.e., $\sum_{i \in N} x_{i}=v(N)$, and individually rational, i.e., $x_{i} \geq v(\{i\})$ for all $i \in N$. The set of imputations is denoted by $I(v)$. The core of a game is the set of imputations that satisfy coalitional rationality and thus are not blocked by any coalition. Formally, given $(N, v)$, the core is the set $C(v)=\left\{x \in \mathbb{R}^{N} \mid x(N)=v(N)\right.$ and $x(S) \geq v(S)$ for all $S \subset N\}$. A game $(N, v)$ is convex if for all $i \in N$ and for all $S \subseteq T \subseteq N \backslash\{i\}$ we have $v(S \cup\{i\})-v(S) \leq v(T \cup\{i\})-v(T)$. The core is an example of set-solution concept. A single-valued solution (or point-solution) on a given set of games $\Gamma$ is a rule $\alpha$ that assigns to each game $(N, v)$ in this set $\Gamma$ an efficient payoff vector $\alpha(v) \in \mathbb{R}^{N}$. Examples of single-valued solutions are the nucleolus and the core-center. Each one of these two solutions selects a core allocation that occupies a "central" position in the core. Although for arbitrary coalitional games these two solutions do not coincide, they do coincide for the particular situation of two-sided Böhm-Bawerk markets.

\subsection{Multi-sided assignment games and the Böhm-Bawerk case}

An $m$-sided assignment problem, denoted by $\left(N^{1}, \ldots, N^{m} ; A\right)$, is given by $m \geq 2$ pairwise disjoint nonempty finite sets (or types) of agents, $N^{1}, \ldots, N^{m}$, and a nonnegative $m$-dimensional matrix $A=\left(a_{E}\right)_{E \in \prod_{k=1}^{m} N^{k}}$. We refer to the $i$ th agent of type $k$ as $i \in N^{k}$, and thus we write, with some abuse of notation, $N^{k}=\left\{1,2, \ldots, n_{k}\right\}$ for all $k \in\{1, \ldots, m\}$. To any $m$-tuple of agents $E=\left(i_{1}, \ldots, i_{m}\right) \in \prod_{k=1}^{m} N^{k}$ it is associated the coalition composed of agents $i_{1} \in N^{1}, \ldots, i_{m} \in N^{m}$, which is called an essential coalition. We also refer to the $m$-tuple $E$ as an essential coalition. Each entry $a_{E} \geq 0$ represents the profit associated to the essential coalition $E$.

A matching among $N^{1}, \ldots, N^{m}$ is a set of essential coalitions, $\mu=\left\{E^{h}\right\}_{h=1}^{n}$ with $\underline{n}=\min _{k \in\{1, \ldots, m\}} n_{k}$, such that any agent belongs at most to one coalition in $\mu$. We denote by $\mathcal{M}\left(N^{1}, \ldots, N^{m}\right)$ the set of all matchings among $N^{1}, \ldots, N^{m}$. An agent $i \in N^{k}$, for some $k \in\{1, \ldots, m\}$, is unmatched under $\mu$ if it does not belong to any of its essential coalitions. A matching $\mu$ is optimal if it maximizes $\sum_{E \in \mu} a_{E}$ in $\mathcal{M}\left(N^{1}, \ldots, N^{m}\right)$. We denote by $\mathcal{M}_{A}^{*}\left(N^{1}, \ldots, N^{m}\right)$ the set of all optimal matchings of $\left(N^{1}, \ldots, N^{m} ; A\right)$. 
For each multi-sided assignment problem $\left(N^{1}, \ldots, N^{m} ; A\right)$, the associated multisided assignment game is the cooperative game $\left(N, \omega_{A}\right)$ with the set of players composed of all agents of all types, $N=\cup_{k=1}^{m} N^{k}$, and characteristic function

$$
\omega_{A}(S)=\max _{\mu \in \mathcal{M}\left(N^{1} \cap S, \ldots, N^{m} \cap S\right)}\left\{\sum_{E \in \mu} a_{E}\right\} \text { for any } S \subseteq N
$$

where the summation over the empty set is zero.

It is known that the core of a multi-sided assignment game, $C\left(\omega_{A}\right)$, coincides with the set of efficient nonnegative vectors $x=\left(x_{11}, \ldots, x_{1 n_{1}} ; \ldots ; x_{m 1}, \ldots, x_{m n_{m}}\right)$, with $x_{k i}$ standing for the payoff to agent $i \in N^{k}$, that satisfy $x(E) \geq a_{E}$ for all $E \in \prod_{k=1}^{m} N^{k}$. As a consequence, the above inequality must be tight if $E$ belongs to some optimal matching, and $x_{k i}=0$ if agent $i \in N^{k}$ is unmatched under some optimal matching.

A particular case of multi-sided assignment games are multi-sided Böhm-Bawerk markets, introduced in Tejada (2010). In these markets, each sector $k \in\{1,2, \ldots, m-$ 1 \} is composed of a nonempty finite set $N^{k}$ of sellers, whereas sector $m$ is composed of a nonempty finite set $N^{m}$ of buyers. Each seller $i_{k} \in N^{k}$ has one good of type $k$ to sell, with a reservation price $c_{k i_{k}}$. Each buyer $i \in N^{m}$ wants to buy a bundle formed by one good of each type, with the singularity that, from her point of view, goods of the same type are homogeneous. We denote by $w_{i}$ the valuation that buyer $i$ places on an arbitrary bundle of $m-1$ goods, one from each sector $N^{1}, \ldots, N^{m-1}$.

An $m$-sided Böhm-Bawerk market (or problem) can be summarized by a pair $(\mathbf{c} ; w)$ where $\mathbf{c}=\left(c_{1}, \ldots, c_{m-1}\right) \in \mathbb{R}^{N_{1}} \times \cdots \times \mathbb{R}^{N_{m-1}}$ are the sellers' valuations and $w=\left(w_{1}, \ldots, w_{n_{m}}\right) \in \mathbb{R}^{N_{m}}$ are the buyers' valuations.

From now on, in order to simplify the analysis of the model, we will assume that valuations of the sellers of each sector are arranged in a nondecreasing way and valuations of the buyers are arranged in a nonincreasing way, i.e.

$c_{k 1} \leq c_{k 2} \leq \cdots \leq c_{k n_{k}}$ for all $k \in\{1,2, \ldots, m-1\}$, and $w_{1} \geq w_{2} \geq \cdots \geq w_{n_{m}}$.

Given an $m$-sided Böhm-Bawerk problem $(\mathbf{c} ; w)$, we denote by $A(\mathbf{c} ; w)$ the $m$-dimensional matrix defined by

$$
a_{E}=\max \left\{0, w_{i_{m}}-\sum_{k=1}^{m-1} c_{k i_{k}}\right\} \text { for all } E=\left(i_{1}, \ldots, i_{m}\right) \in \prod_{k=1}^{m} N^{k}
$$

A consequence of (2) is that

$$
a_{E} \geq a_{E^{\prime}} \Leftrightarrow E \leq E^{\prime} \text { for all } E, E^{\prime} \in \prod_{k=1}^{m} N^{k}
$$


where $E=\left(i_{1}, \ldots, i_{m}\right) \leq E^{\prime}=\left(i_{1}^{\prime}, \ldots, i_{m}^{\prime}\right)$ if and only if $i_{k} \leq i_{k}^{\prime}$ for all $k \in$ $\{1, \ldots, m\}$. When no confusion may arise, we write simply $A$ instead of $A(\mathbf{c} ; w)$.

Then, $\left(N, \omega_{A(\mathbf{c} ; w)}\right)$, where $N$ is composed of all sellers and buyers, is the multisided assignment game — see (1) — associated to the multi-sided Böhm-Bawerk market $(\mathbf{c} ; w)$, which we call the multi-sided Böhm-Bawerk assignment game associated to $(\mathbf{c} ; w)$.

For all $i \in \mathbb{N}$, we introduce the notation $D^{i}=(i, \ldots, i) \in \mathbb{R}^{m}$. By (2), the diagonal matching $\mu=\left\{D^{i} \mid i \in\{1, \ldots, \underline{n}\}\right\}$ is an optimal matching (recall $\underline{n}=$ $\left.\min _{k \in\{1, \ldots, m\}} n_{k}\right)$. Then, the core $C\left(\omega_{A(\mathbf{c} ; w)}\right)$ of $\left(N, \omega_{A(\mathbf{c} ; w)}\right)$ coincides with the following set:

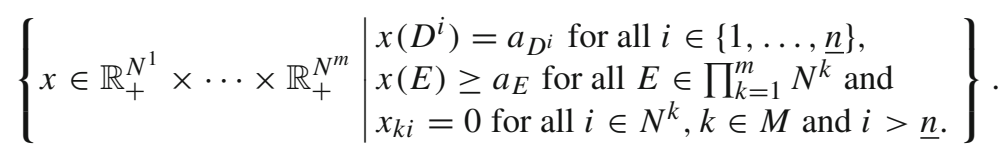

Let us define $r$ as the highest buyer's position that obtains a positive profit when matched with all the sellers in the same position $r$ :

$$
r=\max _{i \in\{1, \ldots, \underline{n}\}}\left\{i \mid a_{D^{i}}>0\right\}
$$

with the convention that $r=0$ if all entries of $A(\mathbf{c} ; w)$ are zero. For each $k \in$ $\{1, \ldots, m\}$, agents $i \in N^{k}$ with $i \in\{1, \ldots, r\}$ are said to be active, while agents $i \in N^{k}$ with $i \in\left\{r+1, \ldots, n_{k}\right\}$ are called inactive. It is not difficult to check that any matching formed by essential coalitions with all agents active is optimal.

From now on, without loss of generality and for the sake of simplicity of the analysis, we add an agent with an arbitrarily high cost if she is a seller, or an agent with an arbitrarily low valuation if she is a buyer, to those sectors $k \in M$ with $n_{k}=r$. A consequence of this assumption is that the existence of the $r+1$ th agent for each sector is ensured.

In Tejada (2010), a new game defined on the set of sectors $M=\{1, \ldots, m\}$ is associated to each multi-sided Böhm-Bawerk assignment game. To this end, for any $S \subseteq M$ let us define $E^{S}=r \mathbf{1}_{S}+(r+1) \mathbf{1}_{M \backslash S} \in \mathbb{R}^{m}$, where, for each $T \subseteq M, \mathbf{1}_{T} \in \mathbb{R}^{m}$ is the vector such that $\mathbf{1}_{T}(k)=1$ if $k \in T$ and $\mathbf{1}_{T}(k)=0$ if $k \notin T$. Notice that, whenever $r>0, E^{S}$ corresponds to the essential coalition formed by the $r$ th agent of each sector in $S$ and the $(r+1)$ th agent of each sector not in $S$.

The following definitions and Theorem 1 below are necessary to obtain our results and can be found in Tejada (2010). We include them for the sake of comprehensiveness of the paper.

Definition 1 Given an $m$-sided Böhm-Bawerk assignment market $(\mathbf{c} ; w)$, the associated sectors game $\left(M, v_{\mathbf{c} ; w}\right)$ is the coalitional game with set of players $M=$ $\{1,2, \ldots, m\}$ composed of all sectors and characteristic function defined, for each $S \subseteq M$, by

$$
v_{\mathbf{c} ; w}(S)=\left\{\begin{array}{cc}
a_{E S} & \text { if } \mathrm{r}>0 \\
0 & \text { if } \mathrm{r}=0
\end{array}\right.
$$


By definition, whenever $r>0$ we have $v_{\mathbf{c} ; w}(M)=a_{D^{r}}>0$ and $v_{\mathbf{c} ; w}(\varnothing)=0$. When no confusion may arise we write $v$ instead of $v_{\mathbf{c} ; w}$. For each $m$-sided BöhmBawerk assignment game $\left(N, \omega_{A(\mathbf{c} ; w)}\right)$, we introduce two more definitions.

Definition 2 The replica operator $\mathcal{R}_{\mathbf{c} ; w}: \mathbb{R}^{M} \longrightarrow \mathbb{R}^{N^{1}} \times \cdots \times \mathbb{R}^{N^{m}}$ is defined by $\mathcal{R}\left(\bar{x}_{1}, \ldots, \bar{x}_{m}\right)=\left(x_{1}, \ldots, x_{m}\right)$, where $x_{k}=(\overbrace{\bar{x}_{k}, \ldots, \bar{x}_{k}}^{r}, \overbrace{0, \ldots, 0}^{n_{k}-r}) \in \mathbb{R}^{N^{k}}$ for all $k \in\{1, \ldots, m\}$.

Notice that $\mathcal{R}_{\mathbf{c} ; w}$ is an injective linear function that maps payoffs of the sectors game to payoffs of the multi-sided Böhm-Bawerk game.

Definition 3 Let $(\mathbf{c} ; w)$ be an $m$-sided Böhm-Bawerk assignment market. Then, the translation vector $t_{\mathbf{c} ; w}=\left(t_{11}, \ldots, t_{1 n_{1}} ; \ldots ; t_{m 1}, \ldots, t_{m n_{m}}\right) \in \mathbb{R}^{N^{1}} \times \cdots \times \mathbb{R}^{N^{m}}$ is defined by

$$
\begin{aligned}
t_{k i} & =\max \left\{0, c_{k r}-c_{k i}\right\} \text { for all } k \in\{1, \ldots, m-1\} \text { and } i \in\left\{1, \ldots, n_{k}\right\}, \\
t_{m i} & =\max \left\{0, w_{i}-w_{r}\right\} \text { for all } i \in\left\{1, \ldots, n_{m}\right\} .
\end{aligned}
$$

A relationship between the core of any multi-sided Böhm-Bawerk assignment game and the core of the corresponding sectors game is stated in the following result.

Theorem 1 Let $(\mathbf{c} ; w)$ be an m-sided Böhm-Bawerk assignment market. Then,

1. $\left(M, v_{\mathbf{c} ; w}\right)$ is convex.

2. $C\left(\omega_{A(\mathbf{c} ; w)}\right)=t_{\mathbf{c} ; w}+\mathcal{R}_{\mathbf{c} ; w}\left(C\left(v_{\mathbf{c} ; w}\right)\right)$.

A consequence of Theorem 1 and (8) is that, for all $x \in C\left(\omega_{A}(\mathbf{c} ; w)\right), k \in M$ and $i \in\{1, \ldots, r\}$, we have $x_{k i}=x_{k r}+t_{k i}$. In the next two sections we show that a statement analogous to Part 2 of Theorem 1 holds for two single-valued solutions that are tightly linked to the core: the nucleolus and the core-center.

It is also worth noting that the relationship of Part 2 of Theorem 1 is not satisfied by every solution concept, e.g. the kernel. ${ }^{1}$ Indeed, on the one hand, since the sectors game is convex, its kernel always reduces to the nucleolus. On the other hand, it is easy to check that the kernel of the 2-sided Böhm-Bawerk assignment game defined by $(\mathbf{c}, w)=(1,1 ; 2,2)$ is the whole core, which is a non-trivial segment. Therefore, the kernel does not satisfy that property.

\section{The nucleolus}

The nucleolus is a single-valued solution for coalitional games introduced by Schmeidler (1969). For any imputation $x$ of $(N, v)$ and any coalition $S \subseteq N$, the excess of coalition $S$ with respect to $x$ is defined by $e_{v}(S, x)=v(S)-x(S)$, and it is a measure

\footnotetext{
${ }^{1}$ The kernel is a solution concept introduced by Davis and Maschler (1965). In the case of a multi-sided assignment game $\left(N, \omega_{A}\right)$, the kernel is the set $K\left(\omega_{A}\right)=\left\{x \in I\left(\omega_{A}\right) \mid s_{i, j}(x)=s_{j, i}(x)\right.$ for any $i \neq j \in$ $N\}$, where $s_{i, j}(x)=\max \{v(S)-x(S) \mid i \in S, j \notin S\}$.
} 
of the satisfaction of coalition $S$ with respect to the allocation $x$. Given an arbitrary game $(N, v)$ with a nonempty set of imputations and an imputation $x \in I(v)$, we define the vector $\lambda(x) \in \mathbb{R}^{2^{N} \backslash\{\emptyset, N\}}$ of excesses of all proper coalitions of $N$ arranged in a non-increasing order, so that those coalitions with a greater complaint occupy the first positions in $\lambda(x)$. That is, $\lambda_{k}(x)=e_{v}\left(S_{k}, x\right)$ for all $k \in\left\{1, \ldots, 2^{n}-2\right\}$ and $\lambda_{k}(x) \geq \lambda_{j}(x)$ if $1 \leq k<j \leq 2^{n}-2$, where $\left\{S_{1}, \ldots, S_{k}, \ldots, S_{2^{n}-2}\right\}$ is the set of all proper coalitions of $N$. The nucleolus of the game $(N, v)$ is the imputation $\eta(N, v)$ (we write $\eta(v)$ for short when no confusion regarding the player set can arise) that minimizes $\lambda(x)$ with respect to the lexicographic order ${ }^{2}$ over the set of imputations. That is, $\lambda(\eta(v)) \leq_{\text {Lex }} \lambda(x)$ for all $x \in I(v)$. It is known that the nucleolus is always a single point and, whenever the core of the game is nonempty, it belongs to the core.

Maschler et al. (1979) give an alternative definition of the nucleolus of a game $(N, v)$ by means of a finite process that iteratively reduces the set of payoffs to a singleton, called the lexicographic center of the game, that is proved to coincide with the nucleolus.

Given a game $(N, v)$ with a nonempty core, let $\mathcal{C}$ be an arbitrary nonempty subset of coalitions of $N$, and consider the algorithm in Maschler et al. (1979) restricted to coalitions in $\mathcal{C}$. This restricted procedure constructs a sequence of coalitions $\Sigma_{\mathcal{C}}^{0} \supseteq$ $\Sigma_{\mathcal{C}}^{1} \supseteq \cdots \supseteq \Sigma_{\mathcal{C}}^{s+1}$ and a sequence of subsets of payoffs $\mathcal{X}_{\mathcal{C}}^{0} \supseteq \mathcal{X}_{\mathcal{C}}^{1} \supseteq \cdots \supseteq \mathcal{X}_{\mathcal{C}}^{s+1}$ such that initially $\alpha_{\mathcal{C}}^{0}=0, \mathcal{X}_{\mathcal{C}}^{0}=C(v), \Sigma_{\mathcal{C}}^{0}=\mathcal{C}$ and $\Delta_{\mathcal{C}}^{0}=\varnothing$ and, for $t \in\left\{0, \ldots, s_{\mathcal{C}}\right\}$, we define recursively

(a) $\alpha_{\mathcal{C}}^{t+1}=\min _{x \in \mathcal{X}_{\mathcal{C}}^{t}} \max _{S \in \Sigma_{\mathcal{C}}^{t}} e_{v}(S, x)$,

(b) $\mathcal{X}_{\mathcal{C}}^{t+1}=\left\{x \in \mathcal{X}_{\mathcal{C}}^{t} \mid \max _{S \in \Sigma_{\mathcal{C}}^{t}} e_{v}(S, x)=\alpha_{\mathcal{C}}^{t+1}\right\}$

(c) $\Sigma_{t+1}^{\mathcal{C}}=\left\{S \in \Sigma_{\mathcal{C}}^{t} \mid e_{v}(S, x)\right.$ is constant on $\left.x \in \mathcal{X}_{\mathcal{C}}^{t+1}\right\}$,

(d) $\Sigma_{\mathcal{C}}^{t+1}=\Sigma_{\mathcal{C}}^{t} \backslash \Sigma_{t+1}^{\mathcal{C}}$ and $\Delta_{\mathcal{C}}^{t+1}=\Delta_{\mathcal{C}}^{t} \cup \Sigma_{t+1}^{\mathcal{C}}$,

where $s_{\mathcal{C}}$ is the last index for which $\Sigma^{s_{\mathcal{C}}} \neq \varnothing$. The set $\mathcal{X}^{s_{\mathcal{C}}+1}$ is called the $\mathcal{C}$-lexicographic center of $(N, v)$. When no confusion is possible we omit the superscript or subscript $\mathcal{C}$. By Maschler et al. (1979), if we take $\mathcal{C}$ to be the set $2^{N}$ of all coalitions, the $2^{N}$-lexicographic center reduces to only one point and it is the nucleolus. For an arbitrary collection $\mathcal{C}$, the procedure is well defined but $\mathcal{X}^{s+1}$ is not necessarily a single point, and even in that case it might not coincide with the nucleolus.

Huberman (1980) shows that it may be that not all coalitions need to be taken into account for the computation of the nucleolus. Given a game $(N, v)$, a nonempty coalition $S \subseteq N$ is an essential coalition in the sense of Huberman (1980) if $v(S)>$ $\sum_{T \in P(S)} v(T)$ for any non-trivial partition $P(S)$ of $S$. It is then proved that only essential coalitions in the sense of Huberman are relevant for the computation of the nucleolus. This means that, if $\mathcal{C} \subseteq 2^{N}$ is the set of essential coalitions in the sense of Huberman for $(N, v)$, then the $\mathcal{C}$-lexicographic center of $(N, v)$ coincides with the nucleolus $\eta(v)$. Moreover, following the proof of Lemma 6.5 in Maschler et al. (1979),

\footnotetext{
2 Given $x, y \in \mathbb{R}^{h}$, we say $x<$ Lex $y$ if there is some $1 \leq i \leq h$ such that $x_{i}<y_{i}$ and $x_{j}=y_{j}$ for $1 \leq j<i$. Also, we say $x \leq_{\text {Lex }} y$ if $x<_{\text {Lex }} y$ or $x=y$.
} 
it is immediate to show that the $\mathcal{C}$-lexicographic center of $(N, v)$ coincides with $\eta(v)$ if and only if

$$
\lambda_{v}^{\mathcal{C}}(\eta(v)) \leq{ }_{\text {Lex }} \lambda_{v}^{\mathcal{C}}(x) \text { for all } x \in I(v)
$$

where $\lambda_{v}^{\mathcal{C}}(x) \in \mathbb{R}^{|\mathcal{C}|}$ is the non-increasingly ordered vector of excesses at $x$ of coalitions in $\mathcal{C}$.

For a given multi-sided assignment game, let us denote by $\mathcal{E}$ the set of individual coalitions and coalitions formed by exactly one agent of each type (what we call essential coalitions in the present paper). As in the bilateral case, it is easy to check that coalitions not in $\mathcal{E}$ are not essential in the sense of Huberman. Notice, however, that it may be the case that an essential coalition has worth zero and in that case it would not be essential in the sense of Huberman. As a consequence of Huberman's result, the $\mathcal{E}$-lexicographic center also reduces to only one point and coincides with the nucleolus. Observe that $|\mathcal{E}|=n_{1} \cdots n_{m}+n_{1}+\cdots+n_{m}$ which is much lower than $\left|2^{N}\right|=2^{n_{1}+\cdots+n_{m}}$.

In this section we show that, in the case of $m$-sided Böhm-Bawerk assignment games, the set of coalitions to be considered in the computation of the nucleolus can be further restricted. To this end we shall use the following lemma (see the "Appendix" for the proof).

Lemma 1 Let $(N, v)$ be a game with a nonempty core and $\mathcal{C}$ a nonempty subset of coalitions of $N$ such that the $\mathcal{C}$-lexicographic center coincides with the nucleolus. Let $\emptyset \neq \mathcal{F} \subseteq \mathcal{C}$ such that, for all $S \in \mathcal{C} \backslash \mathcal{F}$, there is $\mathcal{T}_{S} \subseteq \mathcal{F}$ satisfying that

(i) $e_{v}(S, x) \leq e_{v}(F, x)$ for all $x \in C(v)$ and all $F \in \mathcal{T}_{S}$,

(ii) for any $\emptyset \neq \mathcal{X} \subseteq C(v)$, if $e_{v}(F, x)=e_{v}(F, y)$ for all $x, y \in \mathcal{X}$ and all $F \in \mathcal{T}_{S}$ then $e_{v}(S, x)=e_{v}(S, y)$ for all $x, y \in \mathcal{X}$.

Then, the nucleolus of $(N, v)$ coincides with the $\mathcal{F}$-lexicographic center of $(N, v)$.

The above lemma is now applied to the $m$-sided Böhm-Bawerk assignment game to see that only essential coalitions formed by either one (or $m-1)$ last active agents of some sectors and $m-1$ (or one) first inactive agents of the remaining sectors need to be taken into account to compute the nucleolus. Formally, given $\left(N, \omega_{A(\mathbf{c} ; w)}\right)$ an $m$-sided Böhm-Bawerk assignment game, let $\mathcal{F}^{N}=\mathcal{F}_{m-1}^{N} \cup \mathcal{F}_{1}^{N}$ be the subset of coalitions of $N$ defined as

$$
\mathcal{F}_{m-1}^{N}=\left\{E^{S}|S \subseteq M,| S \mid=m-1\right\}
$$

and

$$
\mathcal{F}_{1}^{N}=\left\{E^{S}|S \subseteq M,| S \mid=1\right\},
$$

where we recall that $E^{S}=r \mathbf{1}_{S}+(r+1) \mathbf{1}_{M \backslash S}$. Observe that $\left|\mathcal{F}^{N}\right|=2 m$, which in general is much lower than $|\mathcal{E}|=n_{1} \cdots n_{m}+n_{1}+\cdots+n_{m}$. 
Theorem 2 Let $(\mathbf{c} ; w)$ be an m-sided Böhm-Bawerk assignment market. Then the nucleolus $\eta\left(\omega_{A(\mathbf{c} ; w)}\right)$ coincides with the $\mathcal{F}^{N}$-lexicographic center of $\left(N, \omega_{A(\mathbf{c} ; w)}\right)$.

Proof We assume $r>0$, since the case $r=0$ is trivial. Consider the $\mathcal{E}$-lexicographic center of $\left(N, \omega_{A}\right)$, which is known to coincide with the nucleolus $\eta\left(\omega_{A}\right)$. It can be easily checked that at step $t=1$ in (9) we obtain $\alpha^{1}=0, \mathcal{X}^{1}=C\left(\omega_{A}\right), \Sigma_{1}=$ $\Delta^{1}=\left\{S \in \mathcal{E} \mid e(S, x)\right.$ is constant in $\left.C\left(\omega_{A}\right)\right\}$ and $\Sigma^{1}=\mathcal{E} \backslash \Sigma_{1}$. Hence, we can start the algorithm of the $\mathcal{E}$-lexicographic center with $\alpha^{0}=0, \mathcal{X}^{0}=C\left(\omega_{A}\right)$ and

$$
\Sigma_{0}=\Delta^{0}=\left\{S \in \mathcal{E} \mid e(S, x) \text { is constant in } C\left(\omega_{A}\right)\right\} \text { and } \Sigma^{0}=\mathcal{E} \backslash \Sigma_{0} .
$$

Thus, the $\mathcal{E}$-lexicographic center of $\left(N, \omega_{A}\right)$ coincides with the $\Sigma^{0}$-lexicographic center of $\left(N, \omega_{A}\right)$ and, therefore, we can consider the latter instead of the former.

Since any essential coalition formed by either only active agents or only inactive agents belongs to some optimal matching, by (5) each such coalition receives a constant payoff in $C\left(\omega_{A}\right)$, and hence, in the above algorithm, $\Sigma^{0}$ is only composed of all essential coalitions containing both active agents and inactive agents, and all singletons formed by one active agent.

Let $x \in C\left(\omega_{A}\right)$ be an arbitrary core allocation. To prove the theorem we will show that $\mathcal{F}^{N}$ satisfies the assumptions of Lemma 1. Thus, let $S \in \Sigma^{0} \backslash \mathcal{F}^{N}$. We distinguish two cases, depending on whether $S$ is an essential coalition or a singleton.

Case 1: $S=E=\left(i_{1}, \ldots, i_{m}\right) \in \prod_{k=1}^{m} N^{k}$.

Consider a set of sectors associated to $E$ defined by $S_{E}=\left\{k \in M \mid 1 \leq i_{k} \leq r\right\}$. By (12), we have $\varnothing \varsubsetneqq S_{E} \varsubsetneqq M$. Due to the non-symmetrical notation of buyers' and sellers' valuations, we must write separately the cases $m \in S_{E}$ and $m \notin S_{E}$. Nevertheless, the proof of the latter case is analogous to the proof of the former and hence we assume $m \in S_{E}$, whereas the case $m \notin S_{E}$ is left to the reader. Let us also denote by $E^{\prime}=\sum_{k \in S_{E}} i_{k} \mathbf{1}_{\{k\}}+(r+1) \mathbf{1}_{M \backslash S_{E}}$ the essential coalition obtained from $E$ by replacing agents of each sector $k \in M \backslash S_{E}$ by the $r+1$ th agent of the same sector. Since $E^{\prime} \leq E$, by (4) we have $a_{E} \leq a_{E^{\prime}}$. We start by proving that, for all $x \in C\left(\omega_{A}\right)$,

$$
e_{\omega_{A}}(E, x) \leq e_{\omega_{A}}\left(E^{S_{E}}, x\right)
$$

Indeed,

$$
\begin{aligned}
& e_{\omega_{A}}(E, x)=a_{E}-\sum_{k=1}^{m} x_{k i_{k}} \leq a_{E^{\prime}}-\sum_{k \in S_{E}} x_{k i_{k}}-\sum_{k \in M \backslash S_{E}} x_{k i_{k}}=a_{E^{\prime}}-\sum_{k \in S_{E}} x_{k i_{k}} \\
& =a_{E^{\prime}}-\left(x_{m r}+\left(w_{i_{m}}-w_{r}\right)\right)-\sum_{k \in S_{E} \backslash\{m\}}\left(x_{k r}+\left(c_{k r}-c_{k i_{k}}\right)\right) \\
& =\max \left\{0, w_{i_{m}}-\sum_{k \in S_{E} \backslash\{m\}} c_{k i_{k}}-\sum_{k \in M \backslash S_{E}} c_{k(r+1)}\right\} \\
& -\left(x_{m r}+\left(w_{i_{m}}-w_{r}\right)\right)-\sum_{k \in S_{E} \backslash\{m\}}\left(x_{k r}+\left(c_{k r}-c_{k i_{k}}\right)\right)
\end{aligned}
$$




$$
\begin{aligned}
& =\max \left\{0, w_{r}-\sum_{k \in S_{E} \backslash\{m\}} c_{k r}-\sum_{k \in M \backslash S_{E}} c_{k(r+1)}+\left(w_{i_{m}}-w_{r}\right)\right. \\
& \left.+\sum_{k \in S_{E} \backslash\{m\}}\left(c_{k r}-c_{k i_{k}}\right)\right\} \\
& -\left(\left(w_{i_{m}}-w_{r}\right)+\sum_{k \in S_{E} \backslash\{m\}}\left(c_{k r}-c_{k i_{k}}\right)\right)-\sum_{k \in S_{E}} x_{k r} \\
& =\max \left\{-\left(\left(w_{i_{m}}-w_{r}\right)+\sum_{k \in S_{E} \backslash\{m\}}\left(c_{k r}-c_{k i_{k}}\right)\right), w_{r}\right. \\
& \left.-\sum_{k \in S_{E} \backslash\{m\}} c_{k r}-\sum_{k \in M \backslash S_{E}} c_{k(r+1)}\right\}-\sum_{k \in S_{E}} x_{k r} \\
& \leq \max \left\{0, w_{r}-\sum_{k \in S_{E} \backslash\{m\}} c_{k r}-\sum_{k \in M \backslash S_{E}} c_{k(r+1)}\right\}-\sum_{k \in S_{E}} x_{k r} \\
& =a_{E} S_{E}-\sum_{k \in S_{E}} x_{k r}=e_{\omega_{A}}\left(E^{S_{E}}, x\right) \text {, }
\end{aligned}
$$

where the second and the third equalities hold by (5) and Theorem 1, the fifth equality holds by adding and subtracting $w_{r}-\sum_{k \in S_{E} \backslash\{m\}} c_{k r}$ to the second term in the maximum operator, and the last inequality holds by (2). We continue by distinguishing two subcases.

Case 1.1: $a_{E^{S_{E}}}>0$.

Recall that by (12), $S_{E} \varsubsetneqq M$. We now prove that, for each $k^{\prime} \notin S_{E}$,

$$
e_{\omega_{A}}\left(E^{S_{E}}, x\right) \leq e_{\omega_{A}}\left(E^{M \backslash\left\{k^{\prime}\right\}}, x\right) .
$$

Before proving (14) observe that, since $x \in C\left(\omega_{A}\right)$, by (5) we have

$$
x\left(E^{M}\right)=\sum_{l \in M} x_{l r}=a_{E^{M}}=w^{r}-\sum_{l \in\{1, \ldots, m-1\}} c_{l r}
$$

and, for each $k \in M \backslash\{m\}$,

$$
x\left(E^{M \backslash\{k\}}\right)=\sum_{l \in M \backslash\{k\}} x_{l r} \geq a_{E^{M \backslash\{k\}}} \geq w^{r}-c_{k(r+1)}-\sum_{l \in M \backslash\{k, m\}} c_{l r} .
$$

Combining the two above expressions we obtain

$$
x_{k r}-\left(c_{k(r+1)}-c_{k r}\right) \leq 0 \text { for all } k \in M \backslash\{m\}
$$


Then, for each $k^{\prime} \notin S_{E}$,

$$
\begin{aligned}
& e_{\omega_{A}}\left(E^{S_{E}}, x\right)=a_{E^{S_{E}}}-\sum_{k \in S_{E}} x_{k r}=w^{r}-\sum_{k \in S_{E} \backslash\{m\}} c_{k r}-\sum_{k \in M \backslash S_{E}} c_{k(r+1)}-\sum_{k \in S_{E}} x_{k r} \\
& =w^{r}-c_{k^{\prime}(r+1)}-\sum_{k \in M \backslash\left\{k^{\prime}, m\right\}} c_{k r}-\sum_{k \in M \backslash\left\{k^{\prime}\right\}} x_{k r}+\sum_{k \in\left(M \backslash\left\{k^{\prime}\right\}\right) \backslash S_{E}}\left(x_{k r}-\left(c_{k(r+1)}-c_{k r}\right)\right) \\
& \leq w^{r}-c_{k^{\prime}(r+1)}-\sum_{k \in M \backslash\left\{k^{\prime}, m\right\}} c_{k r}-\sum_{k \in M \backslash\left\{k^{\prime}\right\}} x_{k r} \\
& \leq \max \left\{0, w^{r}-c_{k^{\prime}(r+1)}-\sum_{k \in M \backslash\left\{k^{\prime}, m\right\}} c_{k r}\right\}-\sum_{k \in M \backslash\left\{k^{\prime}\right\}} x_{k r} \\
& =a_{E^{M \backslash\left\{k^{\prime}\right\}}}-\sum_{k \in M \backslash\left\{k^{\prime}\right\}} x_{k r}=e_{\omega_{A}}\left(E^{M \backslash\left\{k^{\prime}\right\}}, x\right),
\end{aligned}
$$

where the second equality follows from the assumption $a_{E^{S_{E}}}>0$, the third equality is obtained by adding and subtracting $\sum_{k \in\left(M \backslash\left\{k^{\prime}\right\}\right) \backslash S_{E}}\left(x_{k r}+c_{k r}\right)$ and the first inequality holds by (15). Therefore (14) indeed holds.

Next consider the following nonempty subset of $\mathcal{F}_{m-1}^{N}$,

$$
\mathcal{T}_{S}=\left\{E^{M \backslash\{k\}} \mid k \in M \backslash S_{E}\right\} .
$$

From (13) and (14), we have that $e_{\omega_{A}}(E, x) \leq e_{\omega_{A}}(F, x)$ for all $F \in \mathcal{T}_{S}$, which implies that condition (i) of Lemma 1 is satisfied for $S=E$, taking $\mathcal{F}=\mathcal{F}^{N}$. Further, we prove that condition (ii) of Lemma 1 is also satisfied. First of all observe that

$$
\begin{aligned}
x\left(E^{S_{E}}\right) & =\frac{1}{\left|M \backslash S_{E}\right|} \sum_{k \in M \backslash S_{E}} x\left(E^{S_{E}}\right) \\
& =\frac{1}{\left|M \backslash S_{E}\right|} \sum_{k \in M \backslash S_{E}}\left(x\left(E^{M \backslash\{k\}}\right)-x\left(E^{(M \backslash\{k\}) \backslash S_{E}}\right)\right) \\
& =\frac{1}{\left|M \backslash S_{E}\right|}\left(\sum_{k \in M \backslash S_{E}} x\left(E^{M \backslash\{k\}}\right)-\sum_{k \in M \backslash S_{E}} x\left(E^{(M \backslash\{k\}) \backslash S_{E}}\right)\right) \\
& =\frac{1}{\left|M \backslash S_{E}\right|} \sum_{k \in M \backslash S_{E}} x\left(E^{M \backslash\{k\}}\right)-\left(\frac{\left|M \backslash S_{E}\right|-1}{\left|M \backslash S_{E}\right|}\right) x\left(E^{M \backslash S_{E}}\right) \\
& =\frac{1}{\left|M \backslash S_{E}\right|} \sum_{k \in M \backslash S_{E}} x\left(E^{M \backslash\{k\}}\right)-\left(\frac{\left|M \backslash S_{E}\right|-1}{\left|M \backslash S_{E}\right|}\right)\left(a_{E^{M}}-x\left(E^{S_{E}}\right)\right),
\end{aligned}
$$


where the last equality holds since, by (5), $x\left(E^{S_{E}}\right)+x\left(E^{M \backslash S_{E}}\right)=x\left(E^{M}\right)=a_{E^{M}}$. Therefore,

$$
x\left(E^{S_{E}}\right)=\sum_{k^{\prime} \in M \backslash S_{E}} x\left(E^{M \backslash\left\{k^{\prime}\right\}}\right)-\left(\left|M \backslash S_{E}\right|-1\right) a_{E^{M}} .
$$

and

$$
\begin{aligned}
x(E) & =\sum_{k \in S_{E}} x_{k i_{k}}+\sum_{k \in M \backslash S_{E}} x_{k i_{k}}=\sum_{k \in S_{E}} x_{k i_{k}}=x\left(E^{S_{E}}\right)+\sum_{k \in S_{E}} t_{k i_{k}} \\
& =\sum_{k^{\prime} \in M \backslash S_{E}} x\left(E^{M \backslash\left\{k^{\prime}\right\}}\right)-\left(\left|M \backslash S_{E}\right|-1\right) a_{E^{M}}+\sum_{k \in S_{E}} t_{k i_{k}},
\end{aligned}
$$

where the second and third equalities hold by Theorem 1 and the last equality holds by (17). To conclude, by (18), the excess $e_{\omega_{A}}(E, x)$ is an affine combination of the excesses associated to coalitions of $\mathcal{T}_{S}$ :

$$
\begin{aligned}
& e_{\omega_{A}}(E, x)=a_{E}-x(E) \\
& =a_{E}-\sum_{k \in M \backslash S_{E}} x\left(E^{M \backslash\{k\}}\right)+\left(\left|M \backslash S_{E}\right|-1\right) a_{E^{M}}-\sum_{k \in S_{E}} t_{k i_{k}} \\
& =\sum_{k \in M \backslash S_{E}}\left(a_{E^{M \backslash\{k\}}}-x\left(E^{M \backslash\{k\}}\right)\right) \\
& +a_{E}-\sum_{k \in S_{E}} t_{k i_{k}}-\sum_{k \in M \backslash S_{E}} a_{E^{M \backslash\{k\}}}+\left(\left|M \backslash S_{E}\right|-1\right) a_{E^{M}} \\
& =\sum_{k \in M \backslash S_{E}} e_{\omega_{A}}\left(E^{M \backslash\{k\}}, x\right)+c^{S},
\end{aligned}
$$

where the third equality is obtained by adding and subtracting $\sum_{k \in M \backslash S_{E}} a_{E^{M \backslash\{k\}}}$. Therefore, whenever $e_{\omega_{A}}\left(E^{M \backslash\{k\}}, x\right)$ is constant on a subset $\mathcal{X}$ of $C\left(\omega_{A}\right)$ for all $k \in M \backslash S_{E}$, also $e_{\omega_{A}}(E, x)$ is constant on $\mathcal{X}$. Then, as we claimed, the two requirements of Lemma 1 applied to $S=E$ (under the assumptions of Case 1.1) are satisfied for all $x \in C\left(\omega_{A}\right)$, taking $\mathcal{F}=\mathcal{F}^{N}$ and $\mathcal{T}_{S}$ as in (16).

Case 1.2: $a_{E^{S_{E}}}=0$.

In this case, consider the following nonempty subset of $\mathcal{F}_{1}^{N}$,

$$
\mathcal{T}_{S}=\left\{E^{\{l\}} \mid l \in S_{E}\right\}
$$

By (4), we have $w_{A}(F)=0$ for all $F \in \mathcal{T}_{S}$. Then, for each $l \in S_{E}$,

$$
e_{\omega_{A}}\left(E^{S_{E}}, x\right)=-\sum_{k \in S_{E}} x_{k r} \leq-x_{l r}=\omega_{A}\left(E^{l}\right)-x_{l r}=e_{\omega_{A}}\left(E^{l}, x\right),
$$

and hence 


$$
e_{\omega_{A}}(E, x) \leq e_{\omega_{A}}\left(E^{S_{E}}, x\right) \leq e_{\omega_{A}}\left(E^{l}, x\right)
$$

where the first inequality holds by (13). Hence condition (i) of Lemma 1 is satisfied for $S=E$, on the assumptions of Case 1.2, taking $\mathcal{F}=\mathcal{F}^{N}$ and $\mathcal{T}_{S}$ as in (20). Further, condition (ii) of Lemma 1 is also satisfied. Indeed,

$$
\begin{aligned}
e_{\omega_{A}}(E, x) & =a_{E}-x(E)=a_{E}-\sum_{l \in S_{E}} x_{l i_{l}}-\sum_{l \in M \backslash S_{E}} x_{l i_{l}} \\
& =a_{E}-x\left(E^{S_{E}}\right)-\sum_{l \in S_{E}} t_{l i_{l}} \\
& =\sum_{l \in S_{E}} e_{\omega_{A}}\left(E^{\{l\}}, x\right)+a_{E}-\sum_{l \in S_{E}} t_{l i_{l}},
\end{aligned}
$$

where the first equality holds by Theorem 1 and (5), and the last equality holds from $w_{A}(F)=0$ for all $F \in \mathcal{T}_{S}$. Thus, whenever $e_{\omega_{A}}\left(E^{l}, x\right)$ is constant on a subset $\mathcal{X}$ of $C\left(\omega_{A}\right)$ for all $l \in S_{E}$, also $e_{\omega_{A}}(E, x)$ is constant on $\mathcal{X}$. Therefore, the two requirements of Lemma 1 applied to $S=E$ (under the assumptions of Case 1.2) are again satisfied, taking $\mathcal{F}=\mathcal{F}^{N}$ and $\mathcal{T}_{S}$ as in (20).

Case 2: $S=\{i\}$.

By (12), we can assume $i \in N^{l}$, for some $l \in M$ and $i \leq r$. Let $\mathcal{T}_{S}$ be the following singleton of $\mathcal{F}_{1}^{N} \subseteq \mathcal{F}^{N}$,

$$
\mathcal{T}_{S}=\left\{E^{\{l\}}\right\}
$$

By Theorem 1,

$$
e_{\omega_{A}}(\{i\}, x)=-x_{l i}=-t_{l i}-x_{l r}=e_{\omega_{A}}\left(E^{\{l\}}, x\right)-\omega_{A}\left(E^{\{l\}}\right)-t_{l i},
$$

where the last equality holds by adding and subtracting $\omega_{A}\left(E^{\{l\}}\right)$. Therefore properties (i) and (ii) of Lemma 1 are satisfied for $S=\{i\}$, taking $\mathcal{F}=\mathcal{F}^{N}$ and $\mathcal{T}_{S}$ as in (22).

To sum up, the assumptions of Lemma 1 are satisfied for all $S \in \mathcal{E} \backslash \mathcal{F}^{N}$, hence ensuring that the $\mathcal{F}^{N}$-lexicographic center coincides with the nucleolus.

The result in Theorem 2 simplifies the computation of the nucleolus of a multisided Böhm-Bawerk assignment game. As an application, consider a market situation with eight sellers each of whom owns one unit of a homogenous good, eight other sellers each of whom owns one unit of a different homogenous good and ten potential buyers interested in acquiring a bundle formed exactly by one unit of each of the two goods. Let the valuations in this three-sided Böhm-Bawerk assignment market, which translates into a 26-person cooperative game, be

$$
\begin{aligned}
\left(c_{1}, c_{2}, w\right)= & (5,5,7,8, \mathbf{1 0 . 7 5}, 11,12,13 \\
& 5,6,8,9, \mathbf{9 . 2 5}, 10.5,13,13 \\
& 30,28,26,24, \mathbf{2 2}, 21,20,18,17,15)
\end{aligned}
$$


It is straightforward to check that there are five active agents on each side of the market, that is $r=5$, which is marked in bold.

As a result of Theorem 2, in order to calculate the nucleolus of the corresponding coalitional game $\left(N, \omega_{A}(\mathbf{c} ; w)\right)$ with $2^{26}$ coalitions we only have to consider coalitions in $\mathcal{F}^{N}=\mathcal{F}_{1}^{N} \cup \mathcal{F}_{2}^{N}$, where $\mathcal{F}_{1}^{N}=\{(5,6,6),(6,5,6),(6,6,5)\}$ and $\mathcal{F}_{2}^{N}=$ $\{(6,5,5),(5,6,5),(5,5,6)\}$. However, the number of agents is still high, 26, which means that we have to solve several linear programs with 26 variables. The procedure can be simplified further by exploiting the connection between the cores of the multi-sided assignment game and its related sectors game $\left(M, v_{\mathbf{c} ; w}\right)$.

Given the corresponding sectors game $\left(M, v_{\mathbf{c} ; w}\right)$, let us consider the subset of coalitions of $M$ defined by $\mathcal{F}^{M}=\mathcal{F}_{1}^{M} \cup \mathcal{F}_{m-1}^{M}$, where

$$
\mathcal{F}_{m-1}^{M}=\{S \in M,|S|=m-1\}
$$

and

$$
\mathcal{F}_{1}^{M}=\{S \in M,|S|=1\}
$$

and let us see that only coalitions in $\mathcal{F}^{M}$ are needed to obtain the nucleolus of the sectors game.

Theorem 3 Let $(\mathbf{c} ; w))$ be an m-sided Böhm-Bawerk assignment market. Then,

(a) $\eta\left(\omega_{A(\mathbf{c} ; w)}\right)=t_{\mathbf{c} ; w}+\mathcal{R}_{\mathbf{c} ; w}\left(\eta\left(v_{\mathbf{c} ; w}\right)\right)$.

(b) $\eta\left(v_{\mathbf{c} ; w}\right)$ coincides with the $\mathcal{F}^{M}$-lexicographic center of $\left(M, v_{\mathbf{c} ; w}\right)$.

Proof We assume $r>0$, since the case $r=0$ is trivial. For each $x \in C\left(\omega_{A}\right)$, let $\bar{x} \in C(v)$ be the unique core allocation of the sectors game such that $x=t+\mathcal{R}(\bar{x})$. Let $\eta=\eta\left(\omega_{A}\right)$ be the nucleolus of the multi-sided Böhm-Bawerk assignment game. We first prove that $\bar{\eta}=\eta(v)$.

Notice that, for each $S \subseteq M$ and all $x \in C\left(\omega_{A}\right)$, we have

$$
e_{v}(S, \bar{x})=v(S)-\bar{x}(S)=a_{E^{S}}-x\left(E^{S}\right)=e_{\omega_{A}}\left(E^{S}, x\right),
$$

where the second equality holds by Theorem 1 and the definition of the sectors game. Let $\mathcal{H}=\left\{E^{S} \mid \emptyset \neq S \subsetneq M\right\}$. Then, for each $x \in C\left(\omega_{A}\right), x \neq \eta$, it holds that

$$
\lambda_{\omega_{A}}^{2^{N} \backslash\{\emptyset, N\}}(\eta)<_{\text {Lex }} \lambda_{\omega_{A}}^{2^{N} \backslash\{\emptyset, N\}}(x) \Leftrightarrow \lambda_{\omega_{A}}^{\mathcal{H}}(\eta)<_{\text {Lex }} \lambda_{\omega_{A}}^{\mathcal{H}}(x)
$$

The reason is that since $\mathcal{F}^{N}$ satisfies the assumptions of Lemma 1 (see the proof of Theorem 2) and $\mathcal{F}^{N} \subseteq \mathcal{H} \subseteq 2^{N}$, also $\mathcal{H}$ satisfies the assumptions of Lemma 1 and as a consequence the $\mathcal{H}$-lexicographic center of $\left(N, \omega_{A}\right)$ coincides with the nucleolus of $\left(N, \omega_{A}\right)$. Moreover, by (26),

$$
\lambda_{\omega_{A}}^{\mathcal{H}}(\eta)<_{\text {Lex }} \lambda_{\omega_{A}}^{\mathcal{H}}(x) \Leftrightarrow \lambda_{\omega_{A}}^{2^{M} \backslash\{\emptyset, M\}}(\bar{\eta})<_{\text {Lex }} \lambda_{\omega_{A}}^{2^{M} \backslash\{\emptyset, M\}}(\bar{x}) .
$$


The combination of (27) and (28) leads to

$$
\lambda_{\omega_{A}}^{2^{N} \backslash\{\emptyset, N\}}(\eta)<_{\text {Lex }} \lambda_{\omega_{A}}^{2^{N} \backslash\{\emptyset, N\}}(x) \Leftrightarrow \lambda_{v}^{2^{M} \backslash\{\emptyset, M\}}(\bar{\eta})<_{\text {Lex }} \lambda_{v}^{2^{M} \backslash\{\emptyset, M\}}(\bar{x}),
$$

and thus it guarantees that $\lambda_{v}^{2^{M} \backslash\{\emptyset, M\}}(\bar{\eta})<_{\text {Lex }} \lambda_{v}^{2^{M} \backslash\{\emptyset, M\}}(\bar{x})$ for all $\bar{x} \in C(v), \bar{x} \neq \bar{\eta}$, which implies $\bar{\eta}=\eta(v)$ and finishes the proof of statement (a).

To prove statement (b), recall the definitions of $\mathcal{F}^{N}$ and $\mathcal{F}^{M}$ at (10), (11), (24) and (25), and let us consider the mapping $\Psi$ that assigns each coalition of $\mathcal{F}^{M}$ to a unique coalition of $\mathcal{F}^{N}$ in the following way:

$$
\begin{aligned}
\Psi: \mathcal{F}^{M} & \longrightarrow \mathcal{F}^{N} \\
S & \longrightarrow E^{S} .
\end{aligned}
$$

Observe that by the definitions of $\mathcal{F}^{N}$ and $\mathcal{F}^{M}, \Psi$ is bijective. Also, by (26), for all $S \in \mathcal{F}^{M}$ and all $x \in C\left(\omega_{A}\right)$,

$$
e_{v}(S, \bar{x})=e_{\omega_{A}}\left(E^{S}, x\right)
$$

Notice then that

$$
\begin{aligned}
\lambda_{v}^{\mathcal{F}^{M}}(\bar{\eta})<_{\text {Lex }} \lambda_{v}^{\mathcal{F}^{M}}(\bar{x}) & \Leftrightarrow \lambda_{\omega_{A}}^{\mathcal{F}^{N}}(\eta)<_{\text {Lex }} \lambda_{\omega_{A}}^{\mathcal{F}^{N}}(x) \Leftrightarrow \lambda_{\omega_{A}}^{2^{N} \backslash\{\emptyset, N\}}(\eta)<_{\text {Lex }} \lambda_{\omega_{A}}^{2^{N} \backslash\{\emptyset, N\}}(x) \\
& \Leftrightarrow \lambda_{v}^{2^{M} \backslash\{\emptyset, M\}}(\bar{\eta})<_{\text {Lex }} \lambda_{v}^{2^{M} \backslash\{\emptyset, M\}}(\bar{x}),
\end{aligned}
$$

where the first equivalence holds by (30), the second equivalence holds by Theorem 2 and the third equivalence holds by (29).

Consider again the market in the example given in (23) and notice that to obtain the nucleolus of the three-sided Böhm-Bawerk assignment game $\left(N, \omega_{A}\right)$ we essentially have to compute the nucleolus $\eta(v)$ of the sectors game $(M, v)$, which in this case is the three-person game given below:

$$
\begin{array}{lll}
v(\{1\})=a_{566}=0 & v(\{1,2\})=a_{556}=1 \\
v(\{2\})=a_{656}=0.75 & v(\{1,3\})=a_{565}=0.75 & v(\{1,2,3\})=a_{555}=2 . \\
v(\{3\})=a_{665}=0.5 & v(\{2,3\})=a_{655}=1.75 &
\end{array}
$$

It can be checked that $\eta(v)=(0.1250,1.0625,0.8125)$. Then, from part (a) of Theorem 3 we obtain

$$
\begin{aligned}
\eta\left(w_{A}\right)= & (5.875,5.875,3.875,2.875,0.125,0,0,0 \\
& 5.3125,4.3125,2.3125,1.3125,1.0625,0,0,0 \\
& 8.8125,6.8125,4.8125,2.8125,0.8125,0,0,0,0,0) .
\end{aligned}
$$

Finally let us point out that statement (b) in Theorem 3 provides an even better simplification when the sectors game consists of more than three sectors, that is $m>3$, since it guarantees that in the computation of the nucleolus of the sectors game $(M, v)$ 
not all proper coalitions of $M$ have to be considered, but only those of size 1 and $m-1$. Thus, Theorem 3 provides a more efficient algorithm to compute the nucleolus of the sectors game $(M, v)$, since each linear program in the sequence related to the algorithm of the lexicographic center has many less constraints.

\section{The core center}

González-Díaz and Sánchez-Rodríguez (2007) study the core-center (or mass center of the core) of a coalitional game with a nonempty core defined as the mathematical expectation of the uniform probability distribution over the core. Let $U(A)$ denote the uniform distribution defined over the set $A$ and $E(\mathcal{P})$ the expectation of the probability distribution $\mathcal{P}$. Formally, given an arbitrary game $(N, v)$ with a nonempty core, the core-center is defined as $\Phi(v)=E[U(C(v))]$.

The nucleolus of a coalitional game does not necessarily coincide with its mass center. However, for two-sided Böhm-Bawerk assignment markets the nucleolus coincides with the mass center, since it is the midpoint of the core segment. Thus it is natural to ask whether this property extends to multi-sided Böhm-Bawerk assignment markets. To this end it is necessary to simplify the computation of the core-center, since our markets typically have many agents and there are no easy-to-compute formulae that provide the center of mass of a polytope. With this aim in mind, we prove that, like the nucleolus, the core-center $\Phi\left(\omega_{A(\mathbf{c} ; w)}\right)$ of a multi-sided Böhm-Bawerk assignment game $\left(N, \omega_{A(\mathbf{c} ; w)}\right)$ and the core-center $\Phi\left(v_{\mathbf{c} ; w}\right)$ of the corresponding sectors game $\left(M, v_{\mathbf{c} ; w}\right)$ are related by the injective linear mapping $t_{\mathbf{c} ; w}+\mathcal{R}_{\mathbf{c} ; w}(\cdot)$. Our result is proved on the firm basis provided by measure theory (see for instance, Federer 1969).

Theorem 4 Let $(\mathbf{c} ; w)$ be an m-sided Böhm-Bawerk assignment market. Then,

$$
\Phi\left(\omega_{A(\mathbf{c} ; w)}\right)=t_{\mathbf{c} ; w}+\mathcal{R}_{\mathbf{c} ; w}\left(\Phi\left(v_{\mathbf{c} ; w}\right)\right)
$$

Proof We assume $r>0$, since the case $r=0$ is trivial. Let us consider the two metric spaces $\left(\mathbb{R}^{N^{1}} \times \cdots \times \mathbb{R}^{N^{m}}, d^{N}\right)$ and $\left(\mathbb{R}^{M}, d^{M}\right)$, each of them endowed with the corresponding euclidean distance. The dimension $\operatorname{dim}(P)$ of a convex polytope $P$ is the dimension of the minimal affine variety in which $P$ is contained. From Theorem 1 we know that $C\left(\omega_{A}\right) \subseteq \mathbb{R}^{N^{1}} \times \cdots \times \mathbb{R}^{N^{m}}$ and $C(v) \subseteq \mathbb{R}^{M}$ are convex polytopes of the same dimension $\beta=\operatorname{dim}\left(C\left(\omega_{A}\right)\right)=\operatorname{dim}(C(v)) \leq m-1$.

Given an arbitrary metric space $(\Omega, d)$, the diameter of $B \subseteq \Omega$ is defined by $\delta(B)=\sup \{d(x, y) \mid x, y \in B\}$. Let $\delta^{N}$ and $\delta^{M}$ denote the diameters defined on the metric spaces $\left(\mathbb{R}^{N^{1}} \times \cdots \times \mathbb{R}^{N^{m}}, d^{N}\right)$ and $\left(\mathbb{R}^{M}, d^{M}\right)$. We first claim that, for all $B \subseteq C(v) \subseteq \mathbb{R}^{M}$, we have

$$
\sqrt{r} \delta^{M}(B)=\delta^{N}\left(t_{\mathbf{c} ; w}+\mathcal{R}_{\mathbf{c} ; w}(B)\right),
$$


where $r$ is defined in (6). Indeed, if $\bar{x}, \bar{y} \in C(v)$ and $x, y$ are the corresponding elements of $C\left(\omega_{A}\right)$ by Theorem 1 , we have

$$
\begin{aligned}
d^{N}(x, y) & =\left(\sum_{k \in M} \sum_{i \in N^{k}}\left(x_{k i}-y_{k i}\right)^{2}\right)^{1 / 2}=\left(\sum_{k \in M} \sum_{i \in N^{k}, i \leq r}\left(\bar{x}_{k}+t_{k i}-\bar{y}_{k}-t_{k i}\right)^{2}\right)^{1 / 2} \\
& =\left(\sum_{k \in M} r\left(\bar{x}_{k}-\bar{y}_{k}\right)^{2}\right)^{1 / 2}=\sqrt{r} d^{M}(\bar{x}, \bar{y}) .
\end{aligned}
$$

Let $\mu^{N}: \mathbb{R}^{N^{1}} \times \cdots \times \mathbb{R}^{N^{m}} \longrightarrow[0,+\infty)$ and $\mu^{M}: \mathbb{R}^{M} \longrightarrow[0,+\infty)$ be the Hausdorff outer measures of dimension $\beta$ that correspond respectively to $\left(\mathbb{R}^{N^{1}} \times \cdots \times\right.$ $\left.\mathbb{R}^{N^{m}}, d^{N}\right)$ and $\left(\mathbb{R}^{M}, d^{M}\right)$, where we recall that $\beta$ is the dimension of $C\left(\omega_{A}\right)$ and $C(v)$. By definition,

$$
\begin{aligned}
& \mu^{N}(A)=\lim _{\delta \rightarrow 0} \\
& \left(\inf _{\left\{B_{h}\right\}_{h=1}^{+\infty}}\left\{\begin{array}{l|l}
\sum_{h=1}^{+\infty}\left(\delta^{N}\left(B_{h}\right)\right)^{\beta} & \begin{array}{l}
B_{h} \subseteq \mathbb{R}^{N^{1}} \times \cdots \times \mathbb{R}^{N^{m}}, A \subseteq \cup_{h=1}^{+\infty} B_{h} \\
\text { and } \delta^{N}\left(B_{h}\right)<\delta \text { for all } h \geq 1
\end{array}
\end{array}\right\}\right)
\end{aligned}
$$

for any $A \subseteq \mathbb{R}^{N^{1}} \times \cdots \times \mathbb{R}^{N^{m}}$, and

$$
\mu^{M}(A)=\lim _{\delta \rightarrow 0}\left(\inf _{\left\{B_{h}\right\}_{h=1}^{+\infty}}\left\{\begin{array}{l|l}
\sum_{h=1}^{+\infty}\left(\delta^{M}\left(B_{h}\right)\right)^{\beta} & \begin{array}{l}
B_{h} \subseteq \mathbb{R}^{M}, A \subseteq \cup_{h=1}^{+\infty} B_{h} \\
\text { and } \delta^{M}\left(B_{h}\right)<\delta \text { for all } h \geq 1
\end{array}
\end{array}\right\}\right)
$$

for any $A \subseteq \mathbb{R}^{M}$. By Theorem 1 and (32), and using (33) and (34), for all $B \subseteq C(v) \subseteq$ $\mathbb{R}^{M}$, we have

$$
r^{\beta / 2} \mu^{M}(B)=\mu^{N}\left(t_{\mathbf{c} ; w}+\mathcal{R}_{\mathbf{c} ; w}(B)\right) .
$$

With some abuse of notation let us also denote by $\mu^{N}$ and $\mu^{M}$ the restrictions of $\mu^{N}$ and $\mu^{M}$ to the borel sets of $\left(\mathbb{R}^{N^{1}} \times \cdots \times \mathbb{R}^{N^{m}}, d^{N}\right)$ and $\left(\mathbb{R}^{M}, d^{M}\right)$ respectively, which are measures by the Carathéodory Extension Theorem.

For any $H \subseteq \mathbb{R}^{l}$, let $\mathbf{I}_{H}: \mathbb{R}^{l} \longrightarrow \mathbb{R}$ be defined by $\mathbf{I}_{H}(x)=1$ if $x \in H$ and $\mathbf{I}_{H}(x)=0$ if $x \notin H$. By definition of the Lebesgue integral, for every measurable set $B \subseteq C(v) \subseteq \mathbb{R}^{M}$

$$
r^{\beta / 2} \int \mathbf{I}_{B} d \mu^{M}=r^{\beta / 2} \mu^{M}(B)=\mu^{N}\left(t_{\mathbf{c} ; w}+\mathcal{R}_{\mathbf{c} ; w}(B)\right)=\int \mathbf{I}_{t_{\mathbf{c} ; w}+\mathcal{R}_{\mathbf{c} ; w}(B)} d \mu^{N}
$$


where the second equality holds by (35). Moreover, for any simple function $\bar{s}=$ $\sum_{l=1}^{z} \lambda_{l} \mathbf{I}_{B_{l}}: \mathbb{R}^{M} \rightarrow \mathbb{R}$ defined on the measurable sets $B_{1}, \ldots, B_{z} \subseteq C(v) \subseteq \mathbb{R}^{M}$,

$$
\begin{aligned}
r^{\beta / 2} \int \bar{s} d \mu^{M} & =r^{\beta / 2} \int \sum_{l=1}^{z} \lambda_{l} \mathbf{I}_{B_{l}} d \mu^{M}=\sum_{l=1}^{z} \lambda_{l} r^{\beta / 2} \int \mathbf{I}_{B_{l}} d \mu^{M} \\
& =\sum_{l=1}^{z} \lambda_{l} \int \mathbf{I}_{t_{\mathbf{c} ; w}+\mathcal{R}_{\mathbf{c} ; w}\left(B_{l}\right)} d \mu^{N}=\int \sum_{l=1}^{z} \lambda_{l} \mathbf{I}_{t_{\mathbf{c} ; w}}+\mathcal{R}_{\mathbf{c} ; w}\left(B_{l}\right) d \mu^{N} \\
& =\int s d \mu^{N},
\end{aligned}
$$

where $s=\sum_{l=1}^{z} \lambda_{l} \mathbf{I}_{t_{\mathbf{c} ; w}}+\mathcal{R}_{\mathbf{c} ; w}\left(B_{l}\right): \mathbb{R}^{N^{1}} \times \cdots \times \mathbb{R}^{N^{m}} \rightarrow \mathbb{R}$ is the corresponding simple function defined on the measurable sets $t_{\mathbf{c} ; w}+\mathcal{R}_{\mathbf{c} ; w}\left(B_{1}\right), \ldots, t_{\mathbf{c} ; w}+\mathcal{R}_{\mathbf{c} ; w}\left(B_{z}\right) \subseteq$ $C\left(\omega_{A}\right) \subseteq \mathbb{R}^{N^{1}} \times \cdots \times \mathbb{R}^{N^{m}}$ by the constants $\lambda_{1}, \ldots, \lambda_{z}$ respectively. For every measurable nonnegative-valued function $\bar{f}: \mathbb{R}^{M} \rightarrow \mathbb{R}$, by the construction of the Lebesgue integral we obtain

$$
\begin{aligned}
r^{\beta / 2} \int_{C(v)} \bar{f} d \mu^{M}= & \sup _{\substack{\bar{s}: \mathbb{R}^{M} \rightarrow \mathbb{R} \\
\text { simple }}}\left\{r^{\beta / 2} \int \mathbf{I}_{C(v)} \bar{s} d \mu^{M} \mid 0 \leq \bar{s} \leq \bar{f}\right\} \\
= & \sup _{\substack{\bar{s}: \mathbb{R}^{M} \rightarrow \mathbb{R} \\
\text { simple }}}\left\{\int \mathbf{I}_{\left.t_{\mathbf{c} ; w}+\mathcal{R}_{\mathbf{c} ; w}(C(v)) s d \mu^{N} \mid 0 \leq \bar{s} \leq \bar{f}\right\}}\right. \\
= & \sup _{s: \mathbb{R}^{N^{1}} \times \ldots \times \mathbb{R}^{N^{m}} \rightarrow \mathbb{R}}\left\{\int \mathbf{I}_{C\left(\omega_{A}\right)} s d \mu^{N} \mid 0 \leq s \leq f\right\} \\
= & \int_{C\left(\omega_{A}\right)} f d \mu^{N},
\end{aligned}
$$

where the second equality holds by (37), $f: \mathbb{R}^{N^{1}} \times \cdots \times \mathbb{R}^{N^{m}} \rightarrow \mathbb{R}$ denotes the measurable function that is zero elsewhere except in $C\left(\omega_{A}\right)$, where it is defined as the composition of the inverse of the injective linear mapping $t_{\mathbf{c} ; w}+\mathcal{R}_{\mathbf{c} ; w}(\cdot)$ with $\bar{f}$, and the third equality is explained as follows. By Theorem 1 , for any simple function $s: \mathbb{R}^{N^{1}} \times \cdots \times \mathbb{R}^{N^{m}} \rightarrow \mathbb{R}$ such that $0 \leq s \leq f$ there is a simple function $\bar{s}: \mathbb{R}^{M} \rightarrow \mathbb{R}$ such that $0 \leq \bar{s} \leq \bar{f}$ and $\bar{s}(\bar{x})=s(x)$ for all $\bar{x} \in C(v)$ and $x=t_{\mathbf{c} ; w}+\mathcal{R}_{\mathbf{c} ; w}(\bar{x}) \in C\left(\omega_{A}\right)$. Indeed, if $s=\sum_{l=1}^{z} \lambda_{l} \mathbf{I}_{B_{l}}$ for some measurable sets $B_{1}, \ldots, B_{z} \subseteq \mathbb{R}^{N^{1}} \times \cdots \times \mathbb{R}^{N^{m}}$ we can take $\bar{s}=\sum_{l=1}^{z} \lambda_{l} \mathbf{I}_{\bar{B}_{l}}: \mathbb{R}^{M} \rightarrow \mathbb{R}$, where for all $l \in\{1, \ldots, z\}$ we define $\bar{B}_{l}=\left(t_{\mathbf{c} ; w}+\mathcal{R}_{\mathbf{c} ; w}\right)^{-1}\left(B_{l} \cap C\left(\omega_{A}\right)\right) \subseteq \mathbb{R}^{M}$.

It is known that the $\beta$-dimensional Hausdorff measure agrees with the classical area of an embedded submanifold of $\mathbb{R}^{\beta}$, with $\beta \leq m$. Therefore, except for a constant multiplicative factor that coincides with the area of $C\left(\omega_{A}\right)$ and $C(v), d \mu^{N}$ and $d \mu^{M}$ are the probability density functions of the uniform distributions over $C\left(\omega_{A}\right) \subseteq$ 
$\mathbb{R}^{N^{1}} \times \cdots \times \mathbb{R}^{N^{m}}$ and $C(v) \subseteq \mathbb{R}^{M}$ respectively. Hence, by definition of the core-center, for all $k \in M$ and all $i \in N^{\bar{k}}$ such that $1 \leq i \leq r$,

$$
\begin{aligned}
t_{k i}+\Phi_{k}(v) & =t_{k i}+\frac{\int_{C(v)} \bar{x}_{k} d \mu^{M}}{\int_{C(v)} d \mu^{M}}=\frac{r^{\beta / 2} \int_{C(v)}\left(t_{k i}+\bar{x}_{k}\right) d \mu^{M}}{r^{\beta / 2} \int_{C(v)} d \mu^{M}}=\frac{\int_{C\left(\omega_{A}\right)} x_{k i} d \mu^{N}}{\int_{C\left(\omega_{A}\right)} d \mu^{N}} \\
& =\Phi_{k i}\left(\omega_{A(\mathbf{c} ; w)}\right),
\end{aligned}
$$

where the second equality holds by linearity of the Lebesgue integral and the third equality holds by (38), using $\bar{f}(\bar{x})=\bar{x}_{k}+t_{k i}$. The case $i>r$ is trivial since inactive agents obtain a null payoff at any core allocation.

The above result allows us to compute the core-center of the three-sided BöhmBawerk assignment market $\left(N, \omega_{A_{\mathbf{c} ; w}}\right)$ of the example in (23), since we only need to compute the core-center of the three-player associated sectors game $\left(M, v_{\mathbf{c} ; w}\right)$. Observe that in order to obtain the core-center of $C\left(v_{\mathbf{c} ; w}\right)$ we need to compute the area of a bidimensional region embedded in $\mathbb{R}^{3}$. Nevertheless, a well-known result in Measure Theory is that an invertible affine mapping $f: \mathbb{R}^{q} \longrightarrow \mathbb{R}^{q}$ shifts the Lebesgue measure $\mu$ of $\mathbb{R}^{q}$ proportionally to the absolute value of the determinant of $f$, i.e. $\mu(f(A))=|\operatorname{det}(f)| \mu(A)$ for all measurable set $A \subseteq \mathbb{R}^{q}$. Hence, for our purpose of computing the center of mass of $C\left(v_{\mathbf{c} ; w}\right)$ it suffices to calculate the center of mass of the projection of $C(v)$ onto the $\left(\bar{x}_{1}, \bar{x}_{2}\right)$-plane, since $f\left(\bar{x}_{1}, \bar{x}_{2}, \bar{x}_{3}\right)=$ $\left(\bar{x}_{1}, \bar{x}_{2}, 2-\bar{x}_{1}-\bar{x}_{2}-\bar{x}_{3}\right)$ is an invertible affine mapping from $\mathbb{R}^{3}$ to $\mathbb{R}^{3}$ with the

Fig. 1 The $\left(\bar{x}_{1}, \bar{x}_{2}\right)$ projection of the core of the sectors game associated to the three-sided Böhm-Bawerk assignment game of the Example in (23)

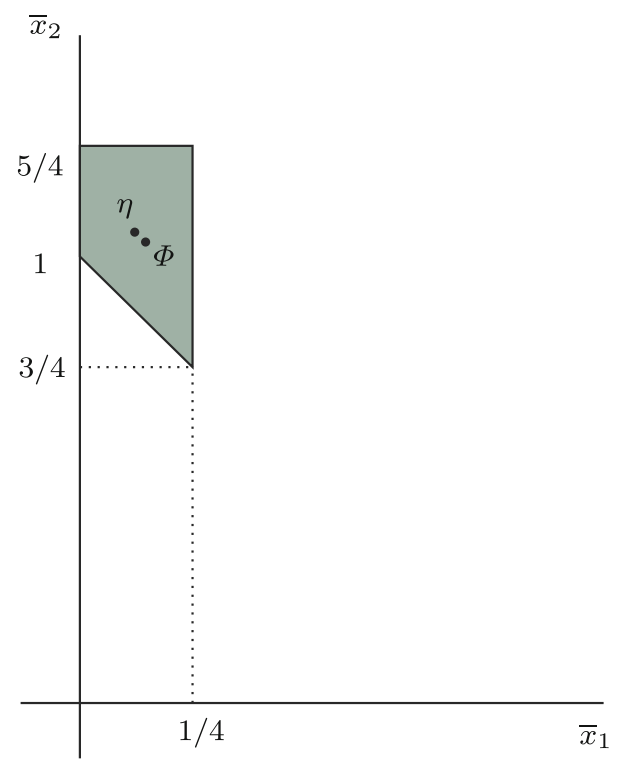


image of $C(v)$ contained in the $\bar{x}_{3}=0$ plane of $\mathbb{R}^{3}$. Notice that this latter computation can be easily carried out using the standard tools of integral calculus in $\mathbb{R}^{2}$, and thus we obtain

$$
\Phi(v)=(0.1389,1.0556,0.8055) .
$$

Figure 1 above depicts the projection of $C(v)$ onto the $\left(\bar{x}_{1}, \bar{x}_{2}\right)$-plane, together with the core-center $\Phi(v)$ and the nucleolus $\eta(v)$ that was obtained at the end of Section 3.

Notice first from $\Phi(v) \neq \eta(v)$ that in general the core-center of a coalitional game differs from the nucleolus, even in the case of convex games. Moreover, the Shapley value (Shapley 1972) of the above sectors game is $(0.1667,1.0417,0.7917)$. Therefore, the Shapley value is in general also different from the core-center for convex games. Finally, as a consequence of Theorems 3 and 4 , from $\Phi(v) \neq \eta(v)$ we deduce that $\Phi\left(\omega_{A}\right) \neq \eta\left(\omega_{A}\right)$ and thus the nucleolus of a multi-sided Böhm-Bawerk assignment market does not coincide in general with the mass center of the core.

Acknowledgments We would like to thank Carles Rafels, two anonymous referees and the associated editor for their very helpful comments, which have helped make the paper much clearer. All remaining errors are our own responsibility. The authors acknowledge the support of the Ministerio de Ciencia e Innovación and FEDER, under grant ECO2008-02344/ECON, and Generalitat de Catalunya, under grant 2009SGR0960. Support from grant Program FPU of Ministerio de Educación of the Spanish Government is also acknowledged.

\section{Appendix}

Proof (of Lemma 1) To simplify the notation, let $\mathcal{X}^{t}, \Sigma_{t}, \Sigma^{t}$ and $\alpha^{t}$ for $t \in$ $\{0,1, \ldots, s, s+1\}$ be the elements of the algorithm leading to the $\mathcal{C}$-lexicographic center of $(N, v)$, where $s$ is the last index for which $\Sigma^{s} \neq \varnothing$, and $\mathcal{X}_{\mathcal{F}}^{t}, \Sigma_{t}^{\mathcal{F}}, \Sigma_{\mathcal{F}}^{t}$ and $\alpha_{\mathcal{F}}^{t}$ for $t \in\left\{0,1, \ldots, s_{\mathcal{F}}, s_{\mathcal{F}}+1\right\}$ be the corresponding elements of the algorithm leading to the $\mathcal{F}$-lexicographic center of $(N, v)$, where $s_{\mathcal{F}}$ is the last index for which $\Sigma_{\mathcal{F}}^{s \mathcal{F}} \neq \varnothing$. We claim that, under the conditions of the lemma, we have $s=s \mathcal{F}$ and, for all $t \in\{0,1, \ldots, s\}, \alpha^{t}=\alpha_{\mathcal{F}}^{t}, \mathcal{X}^{t}=\mathcal{X}_{\mathcal{F}}^{t}$ and $\Sigma^{t} \cap \mathcal{F}=\Sigma_{\mathcal{F}}^{t}$.

We prove this by induction on $t$. The case $t=0$ is trivial by the definition of step $t=0$ in the algorithm given in (9), together with the fact that $\mathcal{F} \subseteq \mathcal{C}$ and thus $\mathcal{F} \cap \mathcal{C}=\mathcal{F}$. Hence, assume that $\alpha^{t}=\alpha_{\mathcal{F}}^{t}, \mathcal{X}^{t}=\mathcal{X}_{\mathcal{F}}^{t}$ and $\Sigma^{t} \cap \mathcal{F}=\Sigma_{\mathcal{F}}^{t}$, for some $t<s$. We shall prove that $\alpha^{t+1}=\alpha_{\mathcal{F}}^{t+1}, \mathcal{X}^{t+1}=\mathcal{X}_{\mathcal{F}}^{t+1}$ and $\Sigma^{t+1} \cap \mathcal{F}=\Sigma_{\mathcal{F}}^{t+1}$.

First we claim that for all $S \in \Sigma^{t}$ there exists $T \in \Sigma^{t} \cap \mathcal{F}$ such that, for all $x \in \mathcal{X}^{t}, e_{v}(S, x) \leq e_{v}(T, x)$. Observe that the inequality holds trivially as an equality if $S \in \mathcal{F}$. Hence, assume that $S \in \mathcal{C} \backslash \mathcal{F}$. By hypothesis of the lemma, there is $\mathcal{T}_{S} \subseteq \mathcal{F}$ such that

$$
e_{v}(S, x) \leq e_{v}(F, x) \text { for all } x \in C(v) \text { and all } F \in \mathcal{T}_{S} \text {, }
$$


and

for any $\emptyset \neq \mathcal{X} \subseteq C(v)$, we have that whenever

$$
\begin{array}{r}
e_{v}(F, x)=e_{v}(F, y) \text { for all } x, y \in \mathcal{X} \text { and all } F \in \mathcal{T}_{S} \text {, then } \\
\qquad e_{v}(S, x)=e_{v}(S, y) \text { for all } x, y \in \mathcal{X} .
\end{array}
$$

If it is the case that $\mathcal{T}_{S} \cap \Sigma^{t}=\emptyset$, then from $\Sigma^{t} \cap \mathcal{F}=\Sigma_{\mathcal{F}}^{t}$ we necessarily have $\mathcal{T}_{S} \subseteq \Delta_{\mathcal{F}}^{t}$, which by construction of (9) implies that, for each $F \in \mathcal{T}_{S}, e_{v}(F, x)$ is constant on $\mathcal{X}_{\mathcal{F}}^{t}=\mathcal{X}^{t}$. Hence, by (40), $e_{v}(S, x)$ is also constant on $\mathcal{X}^{t}$, which contradicts $S \in \Sigma^{t}$. Once the claim is proved, for all $x \in \mathcal{X}^{t}$ it holds that $\max _{S \in \Sigma^{t}} e(S, x) \leq$ $\max _{S \in \Sigma^{t} \cap \mathcal{F}} e(S, x)$ and

$$
\Sigma^{t} \neq \varnothing \Leftrightarrow \Sigma^{t} \cap \mathcal{F} \neq \varnothing
$$

Secondly, for all $x \in \mathcal{X}^{t}$,

$$
\max _{S \in \Sigma^{t}} e_{v}(S, x) \leq \max _{S \in \Sigma^{t} \cap \mathcal{F}} e_{v}(S, x)=\max _{S \in \Sigma_{\mathcal{F}}^{t}} e_{v}(S, x) \leq \max _{S \in \Sigma^{t}} e_{v}(S, x)
$$

where the equality follows from the induction hypothesis and the last inequality from $\Sigma_{\mathcal{F}}^{t}=\Sigma^{t} \cap \mathcal{F} \subseteq \Sigma^{t}$. Hence,

$$
\max _{S \in \Sigma^{t}} e_{v}(S, x)=\max _{S \in \Sigma_{\mathcal{F}}^{t}} e_{v}(S, x)
$$

Thus $\alpha^{t+1}=\min _{x \in \mathcal{X}^{t}} \max _{S \in \Sigma^{t}} e_{v}(S, x)=\min _{x \in \mathcal{X}_{\mathcal{F}}^{t}} \max _{S \in \Sigma_{\mathcal{F}}^{t}} e_{v}(S, x)=\alpha_{\mathcal{F}}^{t+1}$, since $\mathcal{X}^{t}=\mathcal{X}_{\mathcal{F}}^{t}$ also by induction hypothesis.

Now, by (42) and $\mathcal{X}^{t}=\mathcal{X}_{\mathcal{F}}^{t}$ we obtain $\mathcal{X}^{t+1}=\mathcal{X}_{\mathcal{F}}^{t+1}$. Therefore $\Sigma_{t+1} \cap \mathcal{F}=\Sigma_{t+1}^{\mathcal{F}}$ and hence $\Sigma^{t+1} \cap \mathcal{F}=\Sigma_{\mathcal{F}}^{t+1}$.

Finally, by (41) we have $s=s \mathcal{F}$. Thus, since the $\mathcal{C}$-lexicographic center of $(N, v)$ coincides with the nucleolus and $\mathcal{X}^{s+1}=\mathcal{X}_{\mathcal{F}}^{s+1}$, we have that also the $\mathcal{F}$-lexicographic center of $(N, v)$ coincides with the nucleolus.

\section{References}

Davis M, Maschler M (1965) The kernel of a cooperative game. Nav Res Logist Q 12:223-259

Federer H (1969) Geometric measure theory. Springer, New York

González-Díaz J, Sánchez-Rodríguez E (2007) A natural selection from the core of a TU game: the corecenter. Int J Game Theory 36(1):27-46

Huberman G (1980) The nucleolus and the essential coalitions. Anal Optim Syst Lect Notes Control Inf Sci 28:417-422

Kaneko M, Wooders M (1982) Cores of partitioning games. Math Soc Sci 3:313-327

Maschler M, Peleg B, Shapley S (1979) Geometric properties of the kernel, nucleolus, and related solution concepts. Math Oper Res 4:303-338

Núñez M (2004) A note on the nucleolus and the kernel of the assignment game. Int J Game Theory 33(1):55-65 
Núñez M, Rafels C (2005) The Böhm-Bawerk horse market: a cooperative analysis. Int J Game Theory 33(3):421-430

Quint T (1991) The core of an m-sided assignment game. Games Econ Behav 3:487-503

Schmeidler D (1969) The nucleolus of a characteristic function game. SIAM J Appl Math 17:1163-1170

Shapley LS (1972) Cores of convex games. Int J Game Theory 1:11-26

Shapley LS, Shubik M (1972) The assignment game I: the core. Int J Game Theory 1:111-130

Sherstyuk K (1999) Multisided matching games with complementarities. Int J Game Theory 28(4):489-509

Solymosi T, Raghavan TES (1994) An algorithm for finding the nucleolus of assignment games. Int J Game Theory 23:119-143

Solymosi T, Raghavan TES (2001) Assignment games with stable core. Int J Game Theory 30(2):177-185

Stuart HWJr. (1997) The supplier-firm-buyer game and its m-sided generalization. Math Soc Sci 34:21-27

Tejada O, Rafels C (2010) Symmetrically multilateral-bargained allocations in multi-sided assigment markets. Int J Game Theory 39:249-258

Tejada O (2010) Multi-sided Böhm-Bawerk assignment markets: the core (forthcoming in TOP). doi:10.1007/s11750-010-0170-8

Von Böhm-Bawerk E (1923) Positive theory of capital (trans: Smart W). Steckert GE, New York (original publication 1891) 